\title{
Effect of Saline Solution on the Electrical Response of Single Wall Carbon Nanotubes-Epoxy Nanocomposites
}

\author{
Hammad Younes, ${ }^{1}$ Md. Mahfuzur Rahman, ${ }^{1}$ Amal Al Ghaferi, ${ }^{1}$ and Irfan Saadat ${ }^{2}$ \\ ${ }^{1}$ Department of Mechanical and Material Engineering, Masdar Institute of Science and Technology, P.O. Box 54224, Abu Dhabi, UAE \\ ${ }^{2}$ Department of Electrical Engineering and Computer Science, Masdar Institute of Science and Technology, P.O. Box 54224, \\ Abu Dhabi, UAE
}

Correspondence should be addressed to Hammad Younes; hasy193@yahoo.com

Received 9 November 2016; Revised 26 February 2017; Accepted 1 March 2017; Published 4 April 2017

Academic Editor: Evan K. Wujcik

Copyright (C) 2017 Hammad Younes et al. This is an open access article distributed under the Creative Commons Attribution License, which permits unrestricted use, distribution, and reproduction in any medium, provided the original work is properly cited.

\begin{abstract}
The effects of saline solution on the electrical resistance of single wall carbon nanotubes-epoxy nanocomposites have been investigated experimentally. Ultrasonic assisted fabricated $1.0 \%$ and $0.5 \mathrm{~W} / \mathrm{W} \%$ SWCNTs epoxy nanocomposites are integrated into a Kelvin structure by smear cast the nanocomposites on a glass wafer. Four metal pads are deposited on the nanocomposites using the beam evaporator and wires are tethered using soldering. The effect of saline solution on the electrical resistance of the nanocomposites is studied by adding drop of saline solution to the surface of the fabricated nanocomposites and measuring electrical resistance. Moreover, the nanocomposites are soaked completely into $3 \mathrm{wt} . \%$ saline solution and real-time measurement of the electrical resistance is conducted. It is found that a drop of saline solution on the surface of the nanocomposites film increases the resistance by $50 \%$. Furthermore, the real-time measurement reveals a $40 \%$ increase in the resistance of the nanocomposites film. More importantly, the nanocomposites are successfully reset by soaking in DI water for four hours. This study may open the door for using SWCNTs epoxy nanocomposites as scale sensors in oil and gas industry.
\end{abstract}

\section{Introduction}

Scale is caused by the creation of inorganic soluble salts originating from the use of saline solution during the processing in oil and gas production, which deposits at the internal surface of the pipelines under supersaturating conditions. Sodium chloride $(\mathrm{NaCl})$ Calcium Carbonate $\left(\mathrm{CaCO}_{3}\right)$ and Barium Sulphate $\left(\mathrm{BaSO}_{4}\right)$ are considered as the most insoluble inorganic scale that forms during the extraction of oil. The deposition can be observed not only in the pipelines but also in water handling equipment, that is, pumps, valves, and any other parts that interact with water. The deposition of the scale leads to unavoidable damage of equipment, resulting in the suspension of oil operations in order to replace the damaged parts. In the petroleum industry, such interruptions are escorted by extremely high costs.

There have been multiple studies and research conducted on scale monitoring, testing, and optimization of scale inhibitors [1-3]. The current industry practice for managing scale programs relies on the determination of scale inhibitor concentration in the scaling brine and then relates this concentration to program performance. In most cases, scale inhibitors tailored to the specific oil well are used. Often, monitoring and testing for scales require offline sample testing which impacts safety and productivity. Additionally, scale inhibitor residual tests are not ideal because the tests themselves are often time consuming to run, subject to interference from components in the produced water, and specific to the inhibitor used. In general, most of the studies focused on the development and deployment of new sensing materials that enabled monitoring and offline testing [47]. Carbon nanomaterials found to be ideal materials for a new class of sensor systems, which were not possible before. This is due to its 2-D nature (inert, conductive where the conductivity can be modulated when exposed to various ions, molecules, and gases), mechanically very strong yet flexible so that it can be applied to various shapes and forms, in addition to its tolerance to high temperatures (stable till $700^{\circ} \mathrm{C}$ ). 
SWCNTs show unique mechanical, chemical, and physical properties [8-10] which led to a variety of applications, that is, nanofluids [11, 12], scanning probes [13], nanocomposites $[14,15]$, grease [15-17], electron magnetic shielding interface $[18,19]$, and sensors [20-24]. CNT polymer nanocomposites have demonstrated good sensing capability. An et al. [25] fabricated SWCNT/PPY gas sensors for $\mathrm{NO}_{2}$ detecting. The sensitivity of the as-fabricated sensor was about ten times higher than the performance of bare polypyrrole (about $6 \%$ relative resistance change at $200 \mathrm{ppm} \mathrm{NO} \mathrm{NO}_{2}$ ). Zhao and coworkers [26] gave the theoretical analysis of utilizing CNT-metal cluster nanocomposites as a sensing material. CNT-Al clusters were considered in that work. It was shown that adsorption of ammonia causes a charge accumulation in the region between $\mathrm{Al}$ cluster and nanotube. This charge accumulation results in changes in the ionic component of the bonding and shifting the Fermi level of the CNT-Al system. According to these results [26], CNT-metal cluster hybrids could be tailored for detecting different chemical species with high selectivity and sensitivity. Qi et al. [27] built SWCNT- FETs with CVD nanotubes grown across prefabricated metal (Mo) electrode arrays. Noncovalent functionalization of SWCNT with polyethyleneimine (PEI) and Nafion (a polymeric perfluorinated sulfonic acid ionomer) improved sensitivity and selectivity of sensors to $\mathrm{NO}_{2}$ and $\mathrm{NH}_{3}$ gases. PEI coated sensors were capable of detecting less than 1 ppb $\mathrm{NO}_{2}$ while being insensitive to $\mathrm{NH}_{3}$. Nafion-coated devices, on a contrary, showed good sensitivity towards $\mathrm{NH}_{3}$ with no response to $\mathrm{NO}_{2}$.

Single walled carbon nanotubes- (SWCNTs-) epoxy nanocomposites are superior for two reasons: (1) the SWCNTs epoxy nanocomposites are very sensitive to any changes at its surface due to the formation of either covalent or noncovalent bond in between adsorbed molecules and the carbon nanomaterials; (2) epoxy is a very durable thermosetting polymer that has unique physical and chemical stability in corrosive and hostile environments, taken into consideration the temperature in the pipelines and wells is more than $120^{\circ} \mathrm{C}$ along with very high pressure so it is believed that the SWCNTs epoxy nanocomposites will be stable at this hostile conditions.

In this study, SWCNTs epoxy nanocomposites have been successfully fabricated and characterized. In addition, the effects of saline solution on the electrical resistance of SWCNTs epoxy nanocomposites have been investigated experimentally; moreover, the stability of the nanocomposites was studied using thermogravimetric analysis (TGA). Furthermore, the stability of the nanocomposites in the saline solution has been studied by submerging the SWCNTs epoxy nanocomposites in the saline solution for ten hours where the changes of the electrical resistance over a period of time have been monitored. This study may open the door for using SWCNTs epoxy nanocomposites in many applications such as sensors.

\section{Materials and Methods}

Materials. Single wall carbon nanotubes (SWCNTs) were purchased from Sigma-Aldrich, USA, Inc. The chemical surfactant sodium dodecylbenzene sulfonate (NaDDBS) was purchased from Sigma-Aldrich. Epoxy resins and hardener were purchased from west system Inc., Zayed port, Abu Dhabi. Releasing agents, Frekote, were purchased from Logistics Company Limited, Dubai, UAE.

Characterizations. Sonication was performed using a Branson Digital Sonifier, model 450. The electrical conductivity was obtained using Elite 300 Semiautomatic Probe Station \& Keithley 4200-SCS Parameter Analyzer. The microstructures of the samples were probed by Scanning Electron Microscope, FEI quanta 250 ESEM and the FEI Nova NanoSEM 650. Atomic force microscopy data was obtained using (Asylum MFP-3D) deflection $1.5 \mathrm{~V}$, scan speed $0.7 \mathrm{~Hz}$, applied voltage $500 \mathrm{mV}$, in contact mode imaging. Metal contacts were evaporated on the substrates using Temescal BJD-2000 Ebeam Evaporator.

Methods. The used epoxy is a prepolymer consisting of Bisphenol A attached to an epoxide group. NaDDBS surfactant was added to epoxy with a weight ratio of 4 times carbon nanotubes and mixed manually for five minutes. The mixture was then sonicated until the surfactant was dissolved completely and a clear solution was obtained. $0.5 \mathrm{w} / \mathrm{w} \%$ of carbon nanotubes were added to the mixture and sonicated again. The mixture was then mixed manually for $5 \mathrm{~min}$ with hardener by weight ratio of $10: 2.63$. The glass plate was coated with a layer of releasing agent (Frekote) and the mixture was cast on the glass plate. The sample was cured at room temperature for 24 hours.

\section{Results and Discussion}

3.1. Structural Characterization of SWCNTs Epoxy Nanocomposites. To fabricate stable SWCNTs epoxy nanocomposites with reproducible properties is to homogeneously disperse the SWCNTs into the epoxy resin. Strong Van der Waals forces and entanglements of the nanotubes, though, limit homogeneous dispersion unless the tubes can be separated. Ultrasonic dispersion of the SWCNTs into the epoxy resin, along with surfactant, reduces the size of agglomerated material in the epoxy resin and provides the good dispersion. SWCNTs epoxy nanocomposites were prepared by dispersing $0.5 \mathrm{w} / \mathrm{w} \%$ and $1.0 \mathrm{w} / \mathrm{w} \%$ of SWCNTs in Bisphenol A based epoxy resin using ultrahigh tip sonicator.

Figure 1 summarizes the Raman spectra for SWCNTs, SWCNTs epoxy nanocomposites, and epoxy. Raman spectroscopy is considered as one of the most powerful tools for carbon nanotubes characterization. The four main Raman features of CNTs are the radial breathing mode (RBM), the disordered induced D-band, the tangential G band, and the $\mathrm{G}^{\prime}$ band (disorder overtone of $\mathrm{D}$-band). The radial breathing modes (RBM) is found at $270 \mathrm{~cm}^{-1}$, which indicates the presence of SWCNTs. the G band is located near $1600 \mathrm{~cm}^{-1}$; it allows for the determination of the carbon nanotube type, metallic or semiconducting, as the peak is narrow and the G band contains a shoulder by broadening the bottom part; the SWNTs that are used in this work have more semiconducting tubes. The D-band, which is found near $1315.5 \mathrm{~cm}^{-1}$, is due to 

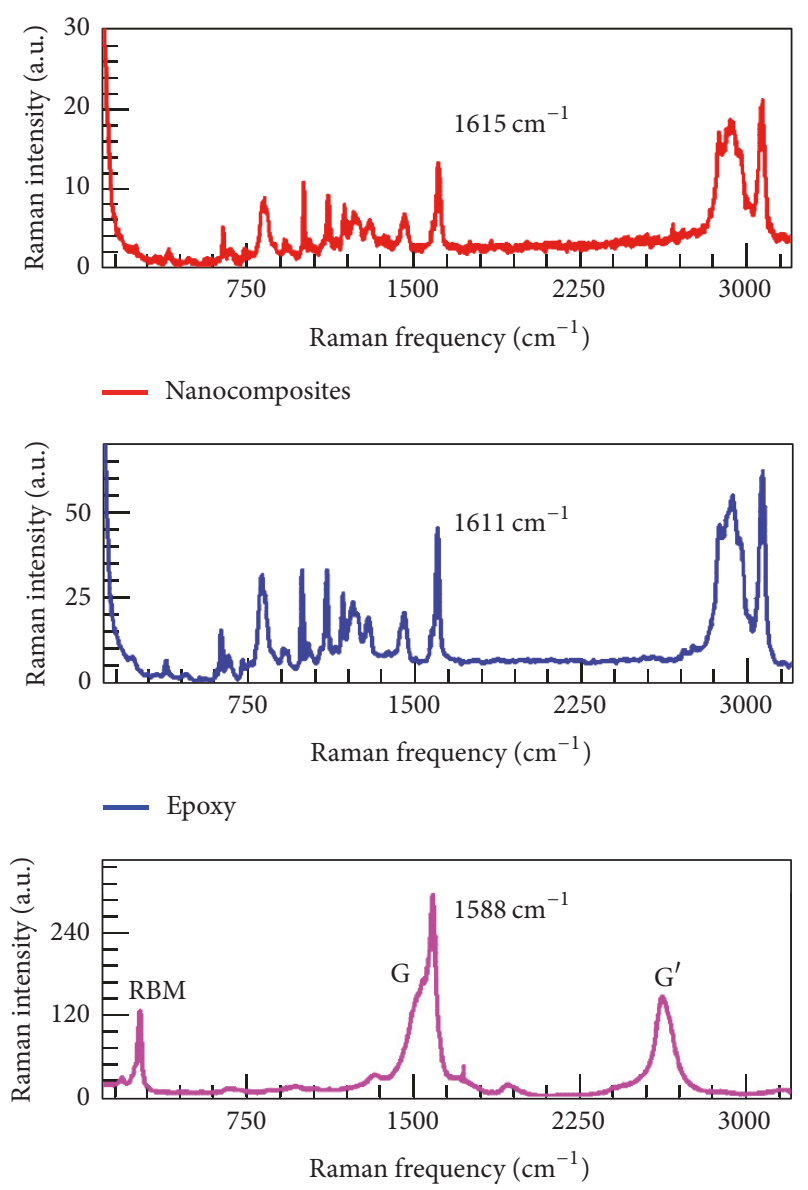

— SWCNTS

FIGURE 1: Raman spectra of SWCNTs epoxy nanocomposites and SWCNTs. For SWCNTs RBM peak appears at $270 \mathrm{~cm}^{-1}$ and for the nanocomposites the intensity of RBM peak was suppressed.

the defects within the carbon nanotubes. This band illustrates the differences between a perfect carbon nanotube (low intensity) and an imperfect carbon nanotube (high intensity). The D-band of the used SWNTs found to be of very low intensity, which tells that SWCNTs that are used in this work have fewer defects. The $\mathrm{G}^{\prime}$ band is in the range of $2620 \mathrm{~cm}^{-1}$. From the Raman spectra of SWCNTs epoxy nanocomposites (Figure 2), it is obvious that the number of Raman signals is increased due to the combined effect of SWCNTs and the epoxy polymer. The $\mathrm{C}-\mathrm{O}-\mathrm{C}$ stretching of the epoxy group was found at $1260 \mathrm{~cm}^{-1}$. The $\mathrm{C}-\mathrm{O}$ stretching (ether groups) and $\mathrm{C}-\mathrm{C}$ stretching are found at $1302 \mathrm{~cm}^{-1}$, and the strong stretching of $\mathrm{C}=\mathrm{C}$ bonds of the aromatic rings is found at $1611 \mathrm{~cm}^{-1}$ [28]. The shift that is seen in the Raman spectra peaks for the SWCNTs epoxy nanocomposites when nanotubes are incorporated into the epoxy resin, particularly the lower-frequency peaks, to higher frequencies, can be attributed to the opening of the nanotube bundles produced by the intercalation of the epoxy [29].

Figure 2 is the SEM images for the nanocomposites showing top and cross-sectional view. It demonstrates the uniform dispersion of SWCNTs in the epoxy polymer forming a network structure which is essential for electrical conductivity. Electrical conduction in such nanocomposites occurs either through tube-tube interaction within the same bundle or between neighboring bundles through their contacts. Therefore, the electrical conductivity depends on the conductivity of the nanotubes themselves and the ability of the electric carriers to tunnel between adjacent nanotubes or adjacent bundles.

Figure 3(a) shows the EDX analysis for the $0.5 \mathrm{wt} \%$ SWCNTs epoxy nanocomposites. As it can be seen from the spectrum, the sample has sodium and chlorine, which indicates the presence of sodium chloride on the surface. The SEM image inset shows many white spots on the surface of the sample, so we selected one big spot and performed the EDX analyses. The red spot is marking one of these big spots.

In order to study the stability of the fabricated nanocomposites in the high temperatures thermogravimetric analysis (TGA) was carried out. Figure 3(b) illustrates the thermograms for pristine SWNTs. As it can be seen thermal degradation of pristine SWCNTs begins at around $500^{\circ} \mathrm{C}$ and all the carbonaceous species are burned off at higher temperature, leaving behind impurities in the SWNTs. However, for SWCNTs epoxy nanocomposites, there was a gradual weight loss as the temperature increased above $150^{\circ} \mathrm{C}$. The weight loss at lower temperatures can be attributed to the evaporation of moisture and surfactant retained by SWCNTs. The rate of differential weight loss is higher in the range of $500-700^{\circ} \mathrm{C}$ because of the burning of carbon species in the aforementioned temperature regime.

Kelvin structure Figure 4 was fabricated by smearing the SWCNTs nanocomposites onto a $2 \times 3$ inches' glass substrate and then metal pads of $15 \mathrm{~nm} \mathrm{Ti}$ and $250 \mathrm{~nm}$ of Al were evaporated on the top of the nanocomposites with Ebeam evaporator and using shadow mask to create four-probe Kelvin structures.

3.2. Electrical Characteristic and Mechanical Properties of CNT Epoxy Nanocomposite. The effect of SWCNTs wt.\% of the electrical resistance of SWCNTs epoxy nanocomposite was studied by measuring IV characteristics of two samples, prepared from $0.5 \mathrm{wt} \%$ (two samples have been prepared; sample $0.5 \mathrm{~A}$ and sample $0.5 \mathrm{~B}$ ) and $1 \mathrm{wt} \% \mathrm{CNT}$ epoxy mixture, respectively. As shown in Figure 5(a), an increase in SWCNTs weight concentration from $0.5 \%$ to $1 \%$ leads to decrease in the electrical resistance from $3.70 E+4$ and $4.01 E+$ 4 to $7.80 E+3$. The CNT epoxy nanocomposite is a $3 \mathrm{D}$ network of dispersed SWCNTs in the epoxy, and the electrical conduction occurs either (1) tube-tube within the structure if the dispersion is highly achieved, (2) SWCNTs- SWCNTs within bundles, or (3) between neighbor bundles through their contacts. Therefore, the electrical conductivity $(\sigma)$ depends on the conductivity of the nanotubes themselves and the ability of the electric carriers to tunnel between adjacent nanotubes or adjacent bundles. In our case, increasing the wt.\% of the SWCNTs increases network density by decreasing the gap between the tubes which creates better contacts and provides more conductive pathways for the charge carriers, which 


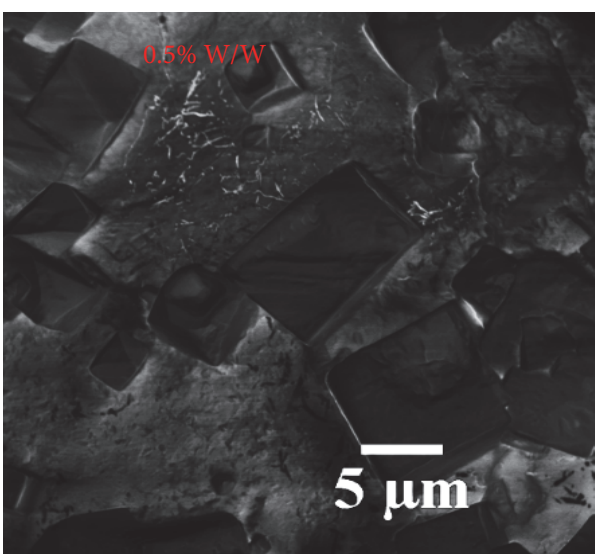

(a)

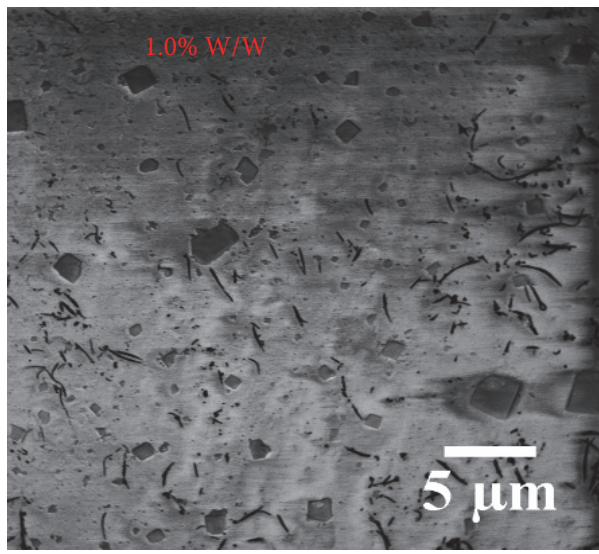

(c)

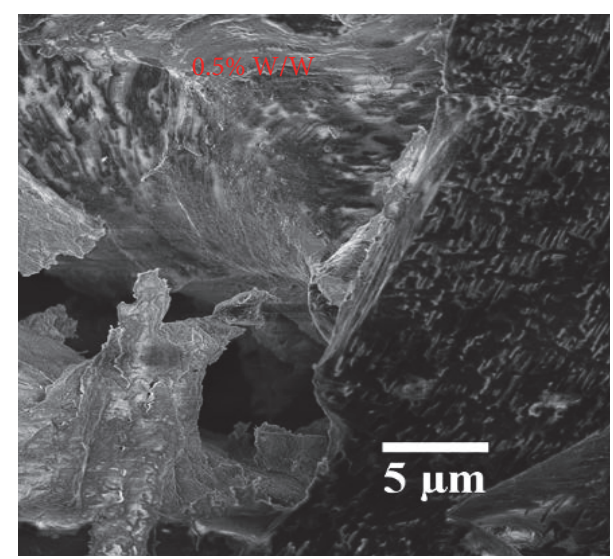

(b)

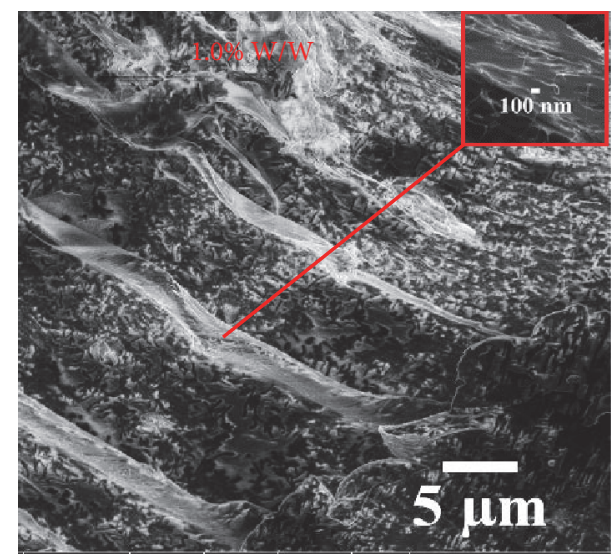

(d)

FIGURE 2: SEM images for SWCNTs epoxy nanocomposites. (a) and (b) are top and cross-sectional view of the 0.5 wt.\% of SWCNTs epoxy and (c) and (d) are the top and cross-sectional view of the $1 \mathrm{w} / \mathrm{w} \%$ of SWCNTs epoxy nanocomposites.

yields an increase in conduction and a decrease in the resistance.

Tensile samples were cut from these large film sheets. Using a razor blade and straight edge, the large films were cut into strips of uniform width. The strips were cut into widths of either $25.4 \mathrm{~mm}$ or $12.7 \mathrm{~mm}$; the width was chosen according to the ASTM Designation: D 882-97 Standard Test Method for Tensile Properties of Thin Plastic Sheeting which states that the test specimen should be of width not less than $5 \mathrm{~mm}$ and not greater than $25.4 \mathrm{~mm}$. Strips were as wide as possible to minimize the effects of edge flaws. The strip length was arbitrarily chosen to be at least $4^{\prime \prime}$, and the initial grip separation was $2^{\prime \prime}$ less than the length of the test strip. Figure 5(b) shows that the SWCNTs epoxy nanocomposites have a tensile strength of $60 \mathrm{Mpa}$; although the tensile strength for neat epoxy is $69 \mathrm{Mpa}$ [30], the results prove that the SWCNTs epoxy nanocomposites are mechanically strong enough to tolerate the hostile environment inside the oil and gas pipelines

3.3. Real-Time Electrical Resistance Measurements. Scale sensing performance of SWCNTs films was characterized by measuring electrical resistance of the sample under different conditions in the following sequence: IV characteristics of the sample were measured in ambient conditions to record the baseline electrical resistance; the sample is then exposed to $3 \mathrm{wt}$ \% of saline solution to analyze the electrical response of CNT epoxy based sensor; and ability to reset the sensor is examined by applying different mechanisms, that is, rinsing or complete immersion in DI water, and the resistance is measured again to confirm sensor recovery.

The resistance of the prepared SWCNTs was measured for the samples just after the preparation and is referred to as resistance of virgin samples while the resistance measured after adding saline is referred to as resistance with saline. Figure 6(a) shows the resistance of the s sample with sweeping voltage from 0 to $1.0 \mathrm{~V}$. The resistance of the sample seems to be very consistent in the swept range. Figure 6(b) shows that adding a drop of saline solution on the surface of the SWCNTs epoxy nanocomposites increases the resistance from $4.50 E+4$ to $6.50 E+4$. Chemical doping can induce strong changes in conductance. Such changes can be easily detected by electron current signals, and these properties make SWCNTs based sensors extremely sensitive to chemical changes.

The real-time sensing measurement in the liquid was done by dipping the sample completely in a beaker with 


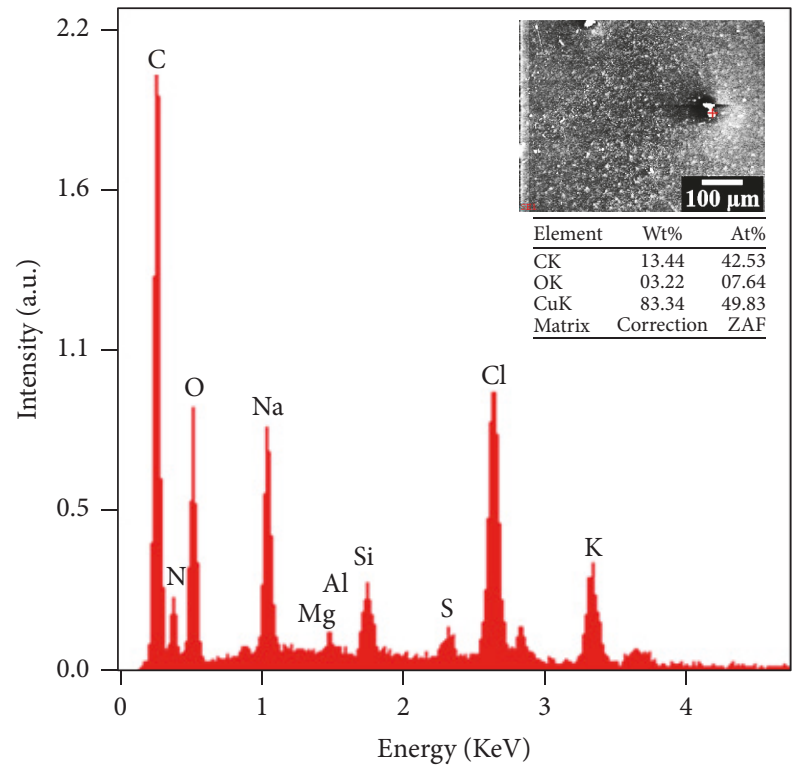

(a)

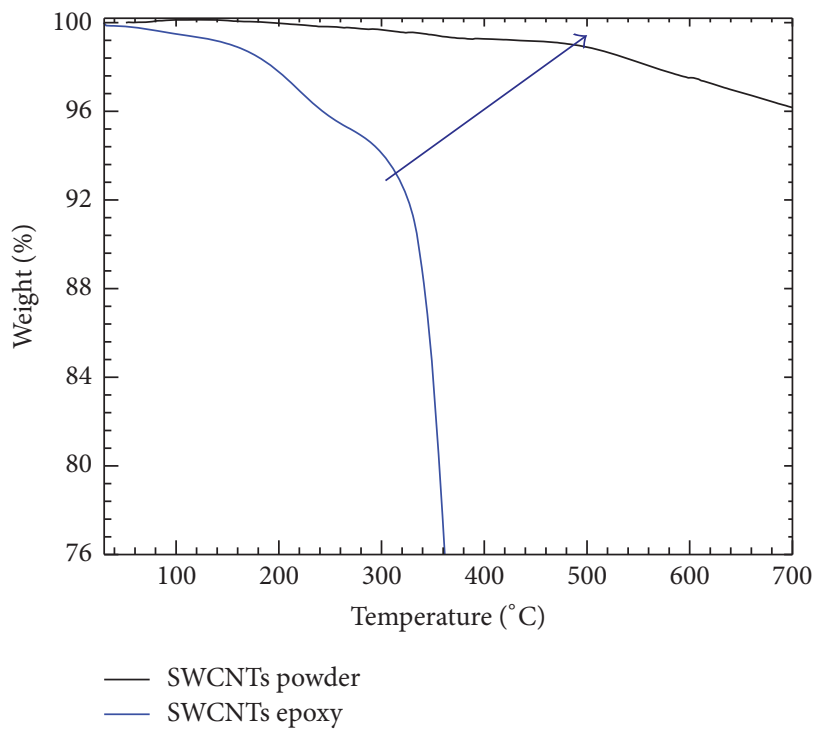

(b)

FIgURE 3: (a) Elemental characterization for saline detection. EDX for SWCNTs epoxy nanocomposites on a glass substrate that has saline molecules at the top of the SWCNTs epoxy nanocomposites surface. (b) Thermogravimetric analysis of pristine SWCNTs and SWCNTs epoxy nanocomposites and SWCNTs epoxy showing weight loss and differential curves at a heating rate of $10^{\circ} \mathrm{C} / \mathrm{min}$ in a nitrogen atmosphere.

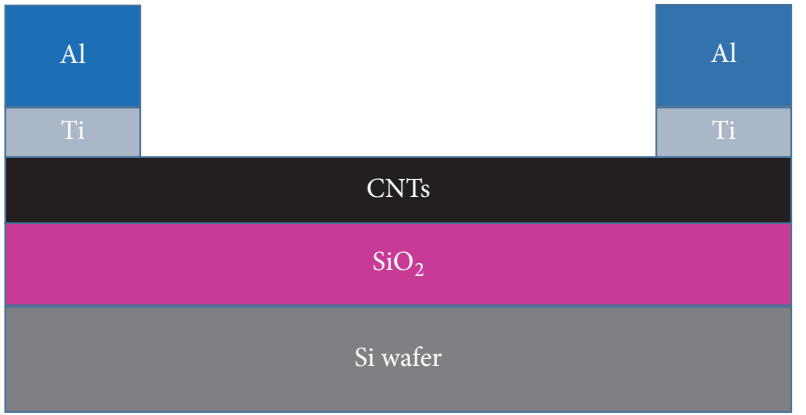

Figure 4: Kelvin structure for SWCNTs epoxy nanocomposites based sensor prepared with smear casting technique with $\mathrm{Ti} / \mathrm{Al}$ metal contacts.

wires coming out of the sample. This approach requires careful insulation of the electrodes in order to ensure that the measured current is that of the CNT films rather than that of the solution itself. The change in resistance is then measured using the Keithley ${ }^{\circledR}$ four-probe setup.

It is very important to have a sensor that can be used for many times. So the ability to reset the sensor is examined by applying different mechanisms, that is, rinsing with DI water or complete immersion in DI water for some time, and the resistance is measured again to confirm the sensor resetting capabilities. The real-time sensing measurement was done for the $0.5 \mathrm{wt} \%$ of SWCNTs epoxy nanocomposites with $3 \mathrm{wt} \%$ of saline as it can be seen that the sample was soaked in $3 \mathrm{wt} . \%$ of saline solution and an in situ measurement for the electrical resistance was done. Figure 7 shows that the sample was recycled almost four times. Soaking the sample in DI water for 3 hours dissolves the saline and removes it from the surface of the sample. The figure shows also the percentage change in the resistance due to the saline or due to the resetting by soaking in water.

Conductive atomic force microscopy C-AFM was carried out for the virgin sample and for the sample with saline solution on the surface. Figure 8 shows that virgin sample (a) has more nanotubes than the sample with saline molecules at the surface (b), which means that C-AFM of SWCNTs epoxy nanocomposites affirm that virgin sample is more conductive than the one with saline and this matches the resistance curve in Figures 8(a) and 8(b).

3.4. Setting and Resetting Process. The setting and resetting processes for $1.0 \mathrm{wt} . \%$ SWCNTs epoxy nanocomposites were studied too. Figure 9 demonstrates one testing cycle for the SWCNTs epoxy nanocomposites based sensor, including the records of baseline resistance of the sample and the electrical response to saline environment.

Figure 9 shows a strong response of the $1.0 \mathrm{wt}$.\% SWCNTs epoxy nanocomposites films to saline solution with the resistance change by $43 \%$. Sensing response can be calculated with a simple formula: $R_{0}$ is the resistance values of the virgin state and $R$ is the resistance after exposure to the saline; the response is as follows:

$$
\delta=\frac{\Delta R}{R_{0}}=\frac{R-R_{0}}{R_{0}}
$$




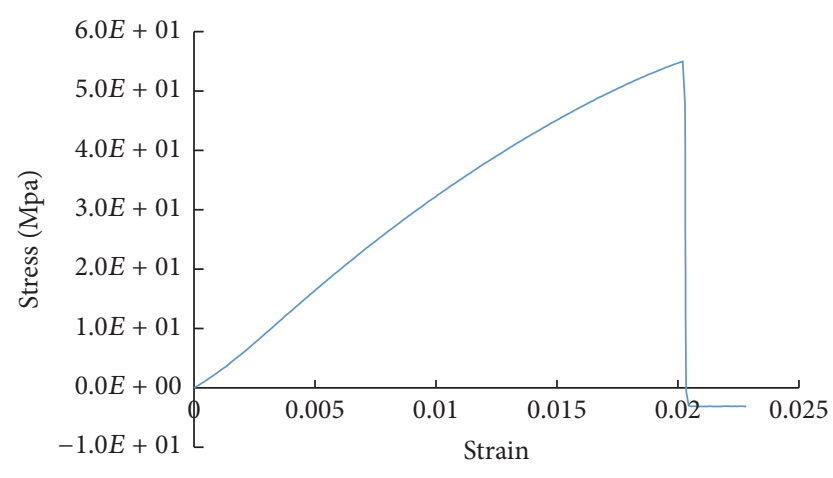

(a)

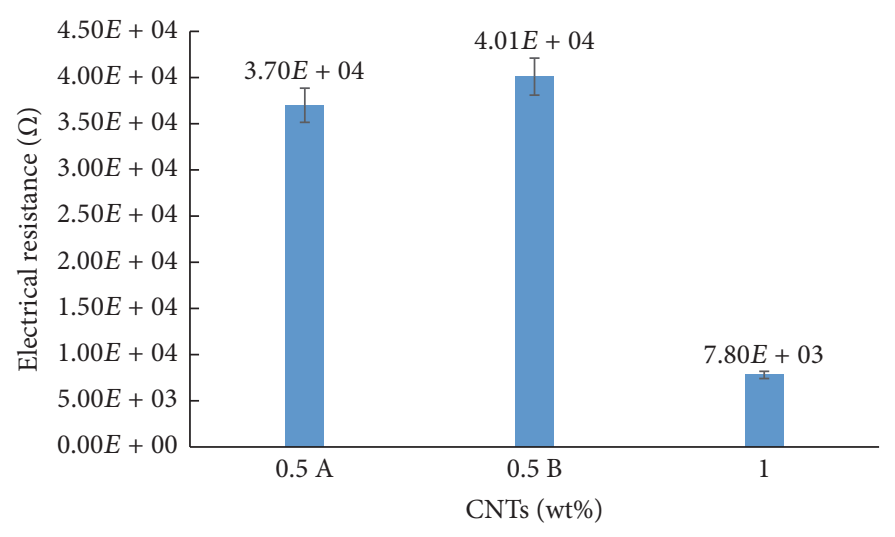

(b)

FIGURE 5: Stress-Strength curves for $0.5 \mathrm{wt} \%$ SWCNTs epoxy nanocomposites. (b) Effect of the SWCNTs wt.\% of the electrical resistance of the SWCNTs epoxy nanocomposites.

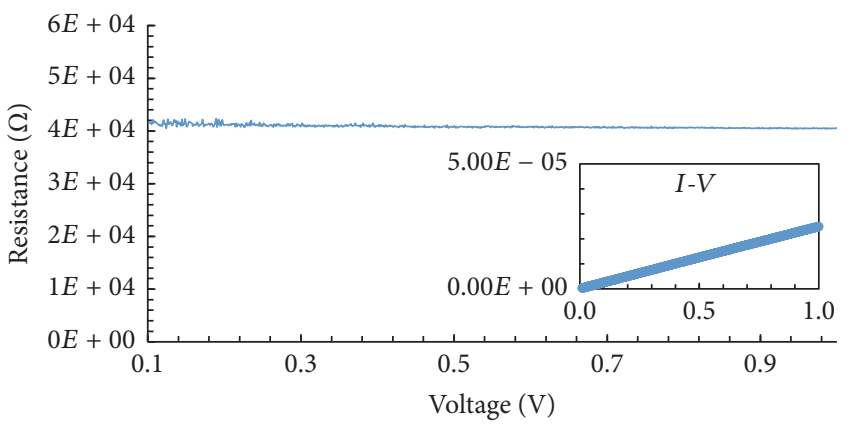

(a)

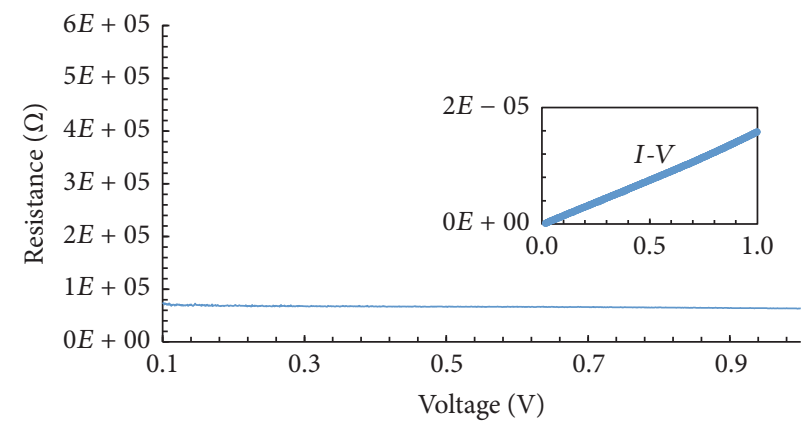

(b)

FIGURE 6: The electrical resistance measurement for (a): 0.5 wt.\% of SWCNTs epoxy nanocomposites virgin sample and (b) 0.5 wt.\% of SWCNTs epoxy nanocomposites in $3 \mathrm{wt} . \%$ saline solution.

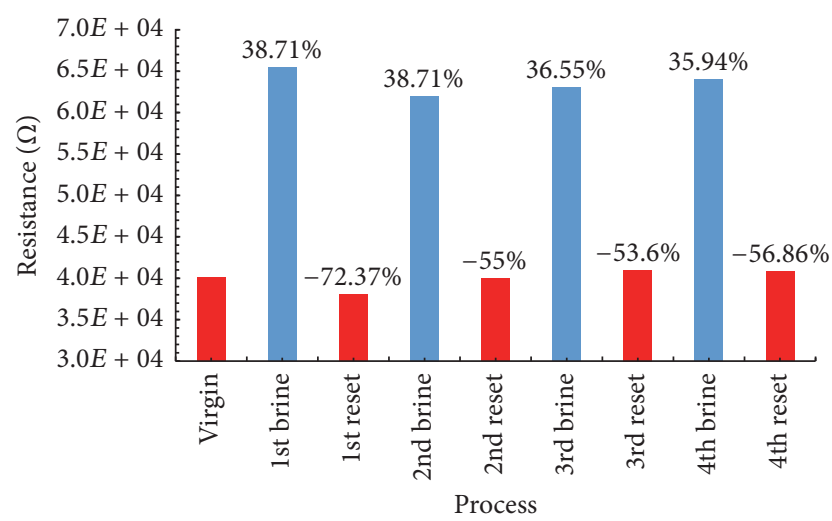

Figure 7: Real-time sensing measurement for $0.5 \mathrm{wt} . \%$ of SWCNTs epoxy nanocomposites in $3 \mathrm{wt} . \%$ saline solution.

According to formula (1), sensitivity of as-fabricated sensor of the $1.0 \mathrm{wt}$. of the SWCNTs epoxy nanocomposites is $16 \%$. In order to check sensor recovery, the sample was washed in DI water and the IV characteristics were measured again. As seen from Figure 9 red curve, rinsing with water did not change the electrical resistance of the sample. This can be attributed to the roughness and the pores that the surface of the nanocomposites has, which trap the salt ions inside and prevent them from being washed out from the surface. Soaking in DI water allows resetting the resistance value by just removing most of the salt ions from the pores and the surface of the nanocomposites and well dissolving them in the DI water.

Multiple tests have been repeated to analyze the sensing performance of the $1.0 \mathrm{wt} \%$ of SWCNTs epoxy nanocomposites based sensor for scale detection. The set/reset performance for $1.0 \mathrm{wt} \%$ of SWCNTs epoxy nanocomposites was quite challenging after the second resetting cycle due to the many pores that the nanocomposites have because of the high concentration of the SWCNTs, but the sensing capability of the SWCNTs nanocomposites along with their stability in hostile environment may open the door for the SWCNTs nanocomposites in many application where the resting of the sensor is not a crucial process.

\section{Conclusion}

SWCNTs epoxy nanocomposites have been successfully fabricated and characterized. The effect of saline solution on the electrical resistance of the nanocomposites due to adding a drop of saline solution to the surface of the fabricated 


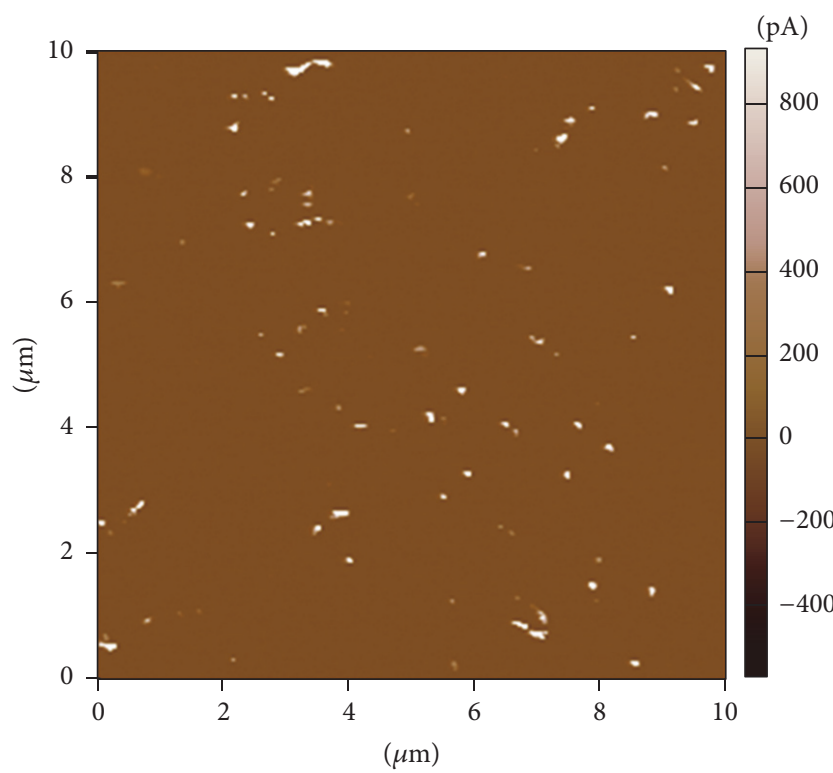

(a)

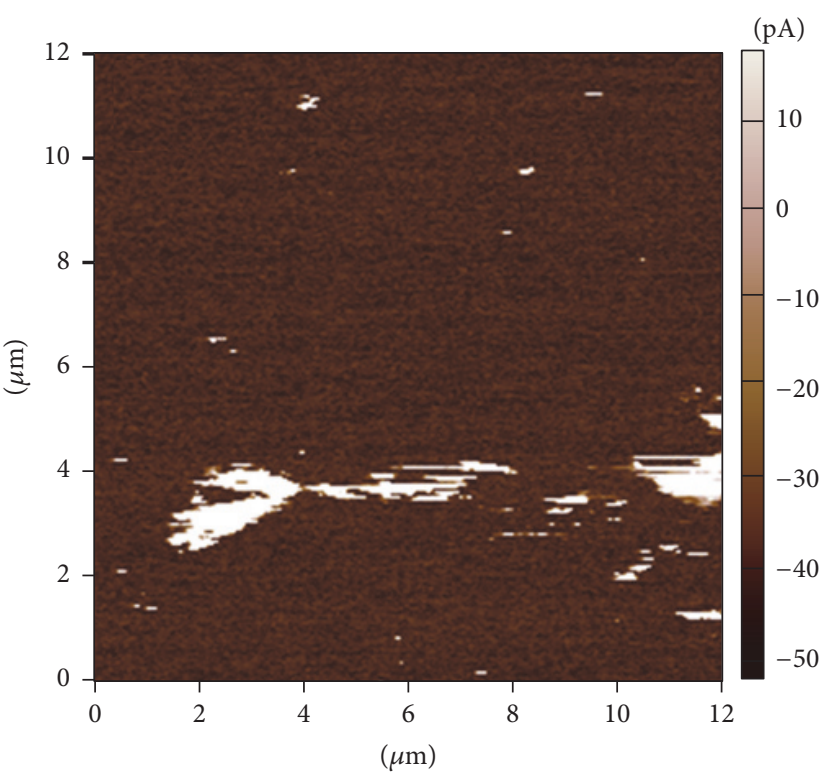

(b)

FIGURE 8: C-AFM for current mapping of 0.5 wt.\% SWCNTs epoxy nanocomposites. (a): virgin sample of SWCNTs epoxy nanocomposites. (b): SWCNTs epoxy nanocomposites sample with saline on the top of the surface.

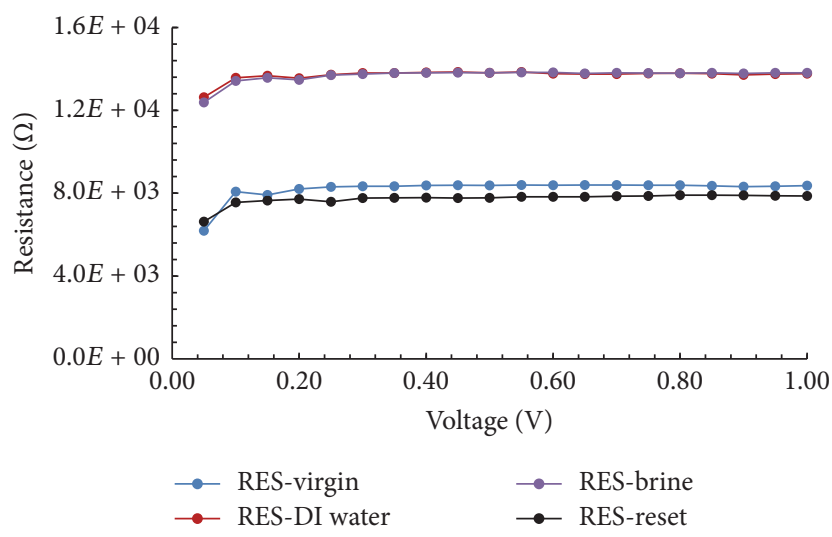

FIGURE 9: Electrical resistance for $1.0 \mathrm{wt} \%$ of SWCNTs epoxy nanocomposites. Blue curve for the virgin sample, purple curve after adding brine solution, red curve after washing with water, and the black one after soaking in water.

nanocomposites was discussed. SWCNTs epoxy nanocomposites were found to be stable at the oil pipelines temperature. It was found that a drop of saline solution on the surface of the $0.5 \mathrm{wt} \%$ nanocomposites film increases the resistance by $50 \%$. Moreover, the real-time measurement showed a $40 \%$ increase in the resistance of the nanocomposites film. More importantly, the nanocomposites were successfully reset once soaked in DI water. Soaking the sample in DI water for 3 hours dissolves the saline and removes it from the surface and the pores of the nanocomposites films. The pores 1.0wt $\%$ of SWCNTs epoxy nanocomposites have made the set/reset performance for $1.0 \mathrm{wt} \%$ of SWCNTs epoxy nanocomposites quite challenging. This study may open the door for using
SWCNTs epoxy nanocomposites as scale sensors in oil and gas industry

\section{Conflicts of Interest}

The authors declare that there are no conflicts of interest regarding the publication of this paper.

\section{Acknowledgments}

The authors would like to acknowledge the entire team of the Masdar Institute Cleanroom and Microscopy Suite for their contribution and support towards this work. The authors would also like to appreciate Petroleum Institute for their partial funding of this research on behalf of the Abu Dhabi National Oil Company (ADNOC), Abu Dhabi, UAE.

\section{References}

[1] D. H. Emmons, G. C. Graham, S. P. Holt, M. M. Jordan, and B. Locardel, "On-site, near-real-time monitoring of scale deposition," in Proceedings of the SPE Annual Technical Conference and Exhibition, October 1999.

[2] M. M. Jordan, "Deployment of real-time scale deposition monitoring equipment to optimize chemical treatment for scale control during stimulation flowback," in Proceedings of the SPE International Oilfield Scale Conference, Society of Petroleum Engineers, May 2008.

[3] H. Al-Matar, J. K. Al-Ashhab, M. Mokhtar, and S. Ridzauddin, "Techniques used to monitor and remove strontium sulfate scale in UZ producing wells," in Proceedings of the Abu Dhabi International Petroleum Exhibition and Conference, Society of Petroleum Engineers, Ed., Abu Dhabi, UAE, 2006. 
[4] R. K. Srivastava, S. Srivastava, T. N. Narayanan et al., "Functionalized multilayered graphene platform for urea sensor," ACS Nano, vol. 6, no. 1, pp. 168-175, 2012.

[5] S. Borini, R. White, D. Wei et al., "Ultrafast graphene oxide humidity sensors," ACS Nano, vol. 7, no. 12, pp. 11166-11173, 2013.

[6] G. Lu, L. E. Ocola, and J. Chen, "Gas detection using low-temperature reduced graphene oxide sheets," Applied Physics Letters, vol. 94, no. 8, Article ID 083111, 2009.

[7] V. Gonçalves, L. Brandão, and A. Mendes, "Development of porous polymer pressure sensors incorporating graphene platelets," Polymer Testing, vol. 37, pp. 127-137, 2014.

[8] A. Bassil, P. Puech, G. Landa et al., "Spectroscopic detection of carbon nanotube interaction with amphiphilic molecules in epoxy resin composites," Journal of Applied Physics, vol. 97, no. 3, Article ID 034303, 2005.

[9] J. G. Park, J. Louis, Q. Cheng et al., "Electromagnetic interference shielding properties of carbon nanotube buckypaper composites," Nanotechnology, vol. 20, no. 41, Article ID 415702, 2009.

[10] T. Inoue, I. Gunjishima, and A. Okamoto, "Synthesis of diameter-controlled carbon nanotubes using centrifugally classified nanoparticle catalysts," Carbon, vol. 45, no. 11, pp. 2164-2170, 2007.

[11] G. Christensen, H. Younes, H. Hong, and G. P. Peterson, "Alignment of carbon nanotubes comprising magnetically sensitive metal oxides by nonionic chemical surfactants," Journal of Nanofluids, vol. 2, no. 1, pp. 25-28, 2013.

[12] H. Younes, G. Christensen, M. Liu, H. Hong, Q. Yang, and Z. Lin, "Alignment of carbon nanofibers in water and epoxy by external magnetic field," Journal of Nanofluids, vol. 3, no. 1, pp. 33-37, 2014.

[13] C. V. Nguyen, Q. Ye, and M. Meyyappan, "Carbon nanotube tips for scanning probe microscopy: fabrication and high aspect ratio nanometrology," Measurement Science and Technology, vol. 16, no. 11, pp. 2138-2146, 2005.

[14] S. Neupane, S. Khatiwada, C. Jaye et al., "Single-walled carbon nanotubes coated by fe2o3 nanoparticles with enhanced magnetic properties," ECS Journal of Solid State Science and Technology, vol. 3, no. 8, pp. M39-M44, 2014.

[15] M. M. Rahman, H. Younes, N. Subramanian, and A. Al Ghaferi, "Optimizing the dispersion conditions of SWCNTs in aqueous solution of surfactants and organic solvents," Journal of Nanomaterials, vol. 2014, Article ID 102621, 11 pages, 2014.

[16] L. Hongtao, J. Hongmin, H. Haiping, and H. Younes, "Tribological properties of carbon nanotube grease," Industrial Lubrication and Tribology, vol. 66, no. 5, pp. 579-583, 2014.

[17] H. Younes, G. Christensen, L. Groven, H. Hong, and P. Smith, "Three dimensional (3D) percolation network structure: key to form stable carbon nano grease," Journal of Applied Research and Technology, vol. 14, no. 6, pp. 375-382, 2016.

[18] Z. Liu, G. Bai, Y. Huang et al., "Reflection and absorption contributions to the electromagnetic interference shielding of single-walled carbon nanotube/polyurethane composites," Carbon, vol. 45, no. 4, pp. 821-827, 2007.

[19] L.-L. Wang, B.-K. Tay, K.-Y. See, Z. Sun, L.-K. Tan, and D. Lua, "Electromagnetic interference shielding effectiveness of carbon-based materials prepared by screen printing," Carbon, vol. 47, no. 8, pp. 1905-1910, 2009.

[20] M. Gautam and A. H. Jayatissa, "Gas sensing properties of graphene synthesized by chemical vapor deposition," Materials Science and Engineering C, vol. 31, no. 7, pp. 1405-1411, 2011.
[21] A. Goldoni, L. Petaccia, S. Lizzit, and R. Larciprete, "Sensing gases with carbon nanotubes: a review of the actual situation," Journal of Physics Condensed Matter, vol. 22, no. 1, Article ID 013001, 2010.

[22] M. Gautam and A. H. Jayatissa, "Ammonia gas sensing behavior of graphene surface decorated with gold nanoparticles," SolidState Electronics, vol. 78, pp. 159-165, 2012.

[23] H. Younes, M. Jelbuldina, L. Tizani et al., "Carbon nanotube inkjet printing based resettable sensor for online scale monitoring," Joural of Nanoscience and Nanotechnology, vol. 17, no. 1, pp. 405-412, 2017.

[24] Y. Hammad, A. G. Amal, and I. Saadat, "Carbon nanostructurebased scale sensors using inkjet printing and casting techniques," in Advances in Carbon Nanostructures, pp. 199-210, InTech Open, 2016.

[25] K. H. An, S. Y. Jeong, H. R. Hwang, and Y. H. Lee, "Enhanced sensitivity of a gas sensor incorporating single-walled carbon nanotube-polypyrrole nanocomposites," Advanced Materials, vol. 16, no. 12, pp. 1005-1009, 2004.

[26] Q. Zhao, M. B. Nardelli, W. Lu, and J. Bernholc, "Carbon nanotube-metal cluster composites: a new road to chemical sensors?" Nano Letters, vol. 5, no. 5, pp. 847-851, 2005.

[27] P. Qi, O. Vermesh, M. Grecu et al., "Toward large arrays of multiplex functionalized carbon nanotube sensors for highly sensitive and selective molecular detection," Nano Letters, vol. 3, no. 3, pp. 347-351, 2003.

[28] K. E. Chike, M. L. Myrick, R. E. Lyon, and S. M. Angel, "Raman and near-infrared studies of an epoxy resin," Applied Spectroscopy, vol. 47, no. 10, pp. 1631-1635, 1993.

[29] D. Puglia, L. Valentini, and J. M. Kenny, "Analysis of the cure reaction of carbon nanotubes/epoxy resin composites through thermal analysis and Raman spectroscopy," Journal of Applied Polymer Science, vol. 88, no. 2, pp. 452-458, 2003.

[30] Y. Shimamura, K. Oshima, K. Tohgo et al., "Tensile mechanical properties of carbon nanotube/epoxy composite fabricated by pultrusion of carbon nanotube spun yarn preform," Composites Part A: Applied Science and Manufacturing, vol. 62, pp. 32-38, 2014. 

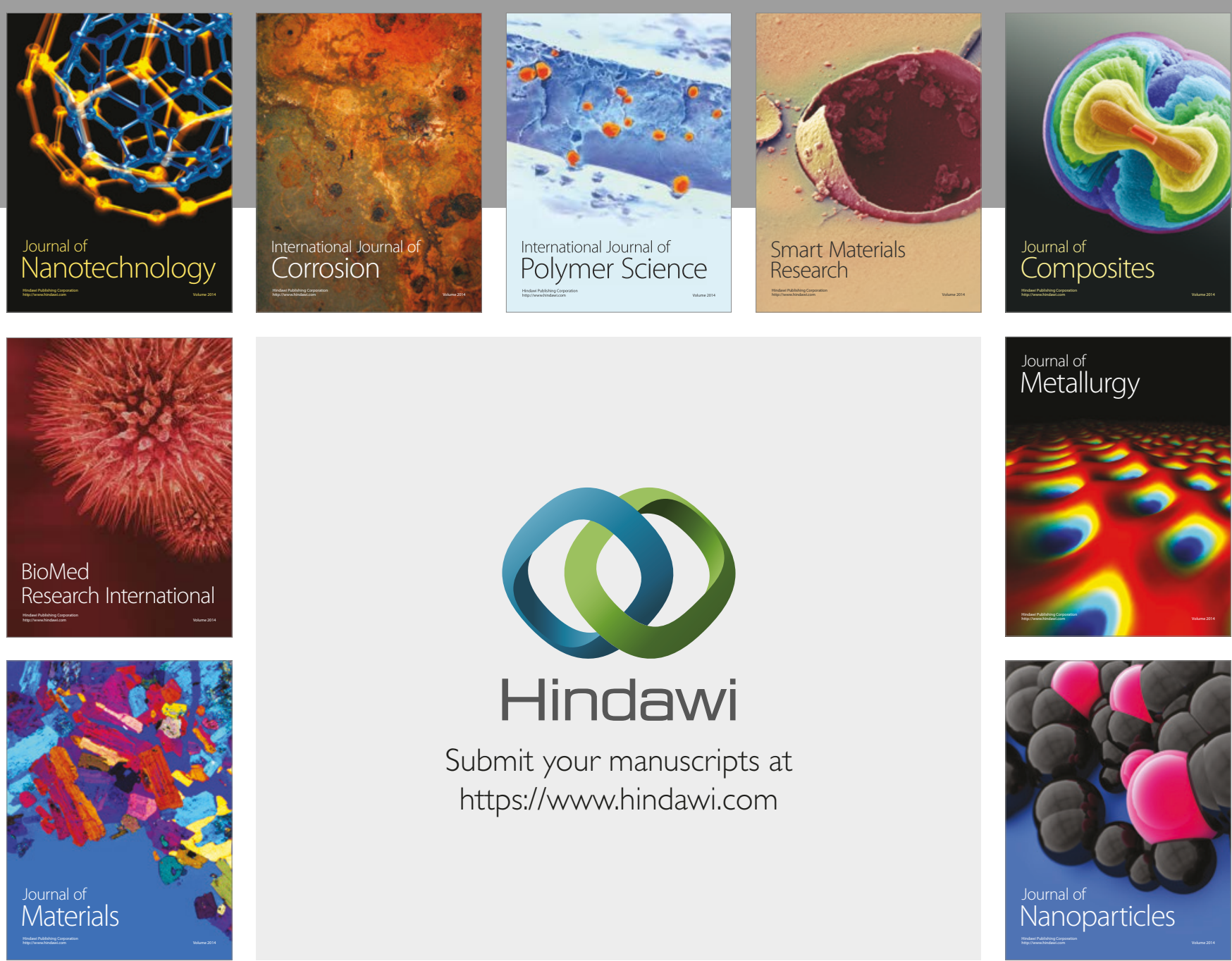

\section{Hindawi}

Submit your manuscripts at

https://www.hindawi.com
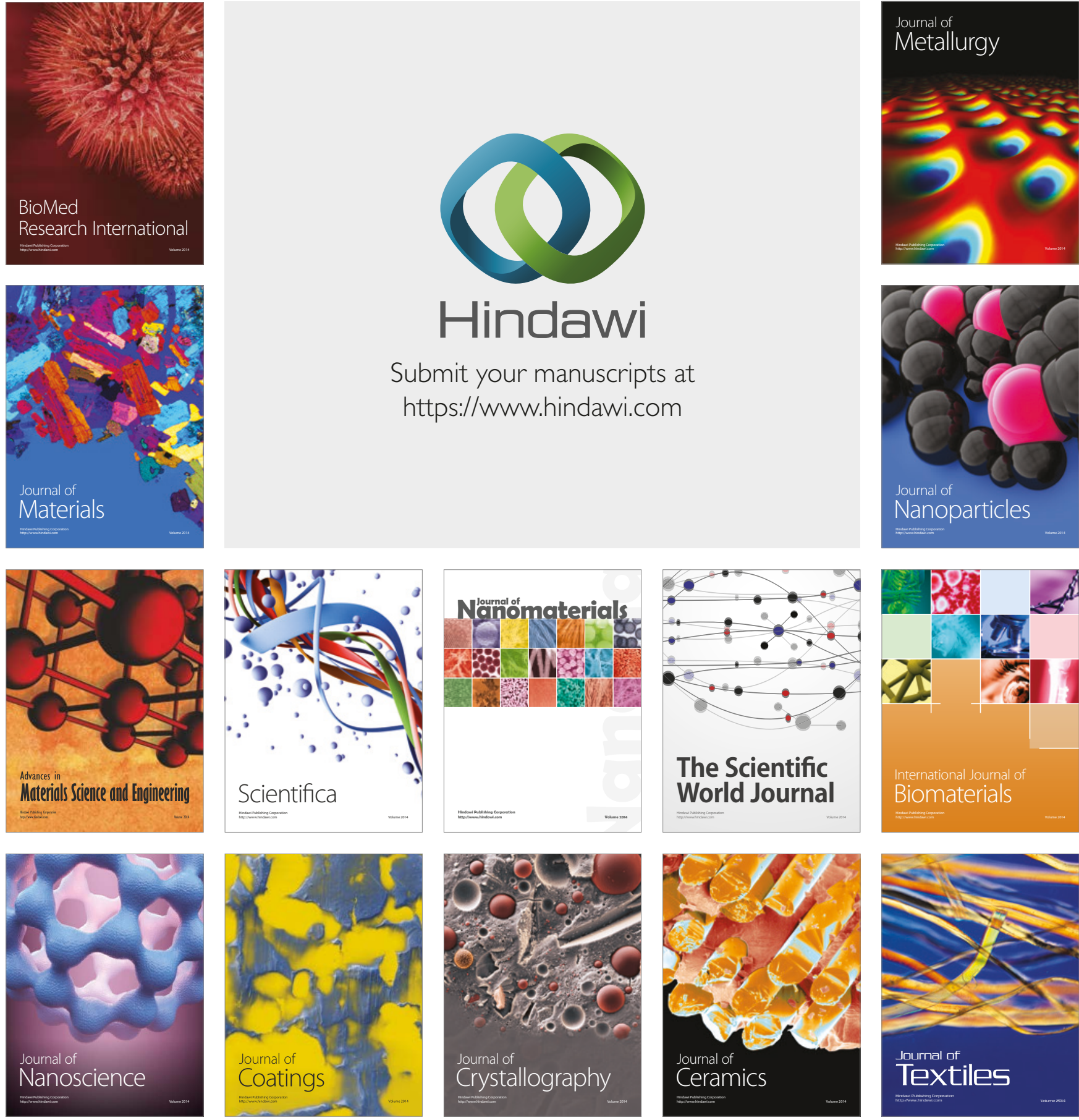

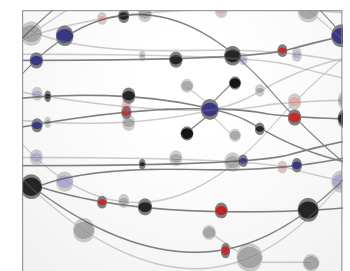

The Scientific World Journal
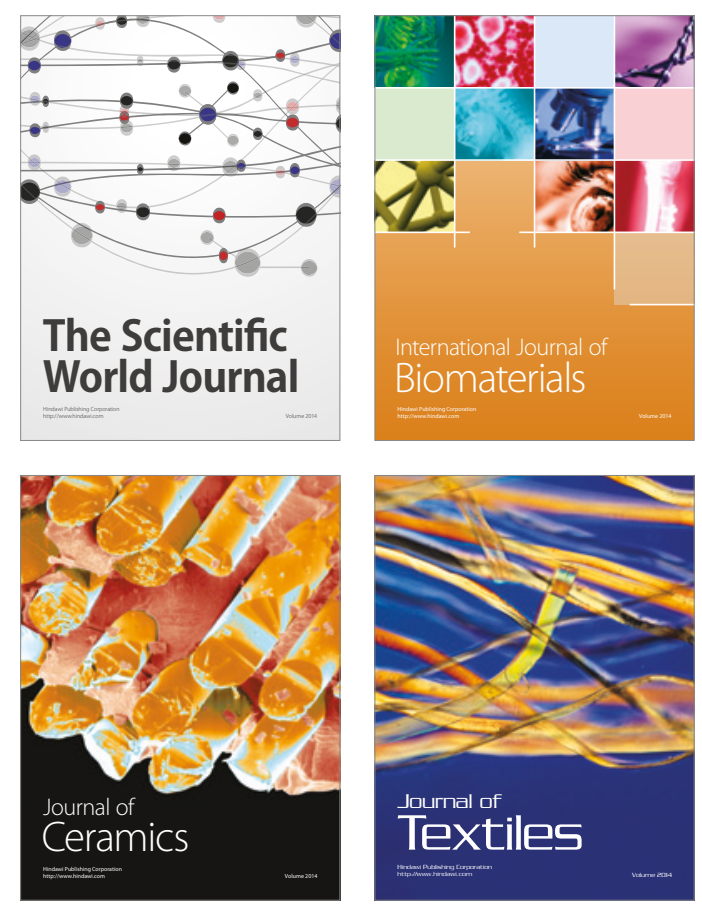